\title{
The neutrophil elastase inhibitor, sivelestat, attenuates sepsis-related kidney injury in rats
}

\author{
GUOFU LI, JIA JIA, KAIQIANG JI, XIAOYING GONG, RUI WANG, \\ XIAOLI ZHANG, HAIYUAN WANG and BIN ZANG \\ Department of Critical Care Medicine, Shengjing Hospital of \\ China Medical University, Shenyang, Liaoning 110004, P.R. China
}

Received November 2, 2015; Accepted June 24, 2016

DOI: $10.3892 / \mathrm{ijmm} .2016 .2665$

\begin{abstract}
Sepsis-induced acute kidney injury (AKI) represents a major cause of mortality in intensive care units. Sivelestat, a selective inhibitor of neutrophil elastase (NE), can attenuate sepsis-related acute lung injury. However, whether sivelestat can preserve kidney function during sepsis remains unclear. In this study, we thus examined the effects of sivelestat on sepsisrelated AKI. Cecal ligation and puncture (CLP) was performed to induce multiple bacterial infection in male Sprague-Dawley rats, and subsequently, 50 or $100 \mathrm{mg} / \mathrm{kg}$ sivelestat were administered by intraperitoneal injection immediately after the surgical procedure. In the untreated rats with sepsis, the mean arterial pressure (MAP) and glomerular filtration rate (GFR) were decreased, whereas serum blood urea nitrogen (BUN) and neutrophil gelatinase-associated lipocalin (NGAL) levels were increased. We found that sivelestat promoted the survival of the rats with sepsis, restored the impairment of MAP and GFR, and inhibited the increased BUN and NGAL levels; specifically, the higher dose was more effective. In addition, sivelestat suppressed the CLP-induced macrophage infiltration, the overproduction of pro-inflammatory mediators (tumor necrosis factor- $\alpha$, interleukin-1 $\beta$, high-mobility group box 1 and inducible nitric oxide synthase) and serine/threonine kinase (Akt) pathway activation in the rats. Collectively, our data suggest that the inhibition of NE activity with the inhibitor, sivelestat, is beneficial in ameliorating sepsis-related kidney injury.
\end{abstract}

\section{Introduction}

Sepsis is a systemic response to invasive microbial infection or severe tissue damage, resulting in tissue necrosis, multi-organ failure and death (1). After being triggered by overwhelming initial stimuli, neutrophils and macrophages can produce exces-

Correspondence to: Dr Guofu Li, Department of Critical Care Medicine, Shengjing Hospital of China Medical University, 36 Sanhao Street, Shenyang, Liaoning 110004, P.R. China

E-mail: guofli13@126.com

Key words: acute kidney injury, sepsis, sivelestat, neutrophil elastase, Akt signaling pathway sive pro-inflammatory mediators, leading to a dysregulated immune response that characterizes sepsis (2). The dominant causes of acute kidney injury (AKI) in hospitalized patients are sepsis and septic shock (3). Lipopolysaccharide (LPS), known as the major component of the outer membrane of Gram-negative bacteria, is a potent activator for acute inflammatory response in sepsis (4), and LPS signaling is initiated by activation of Toll-like receptor 4 (TLR4) (5). Therefore, the blockade of TLR4 signaling has been considered as a promising therapeutic approach for sepsis $(6,7)$ and draws intensive attention from scientists and clinicians worldwide. However, a recent randomized double-blind controlled trial revealed that the application of a specific TLR4 antagonist failed to reduce sepsis-induced mortality (8). Thus, the further identification of critical mediators and molecular targets in sepsis is required. The developmetn of effective therapeutic approaches is urgenly required.

Reportedly, following bacterial infection, the massive recruitment and activation of neutrophils are presented with the extracellular release of neutrophil elastase (NE). NE is a serine protease that propagates persistent neutrophilic inflammation by accelerating pro-inflammatory cytokine production $(9,10)$. By using mouse models of ischemic AKI, Zager et al observed a marked increase in renal cortical/isolated proximal tubule NE mRNA levels and a decrease in NE protein levels (11). Their study suggested that the downregulated protein expression of renal NE correlated with the upregulation of endogenous $\alpha$-1-antitrypsin, which has protease inhibitor activity (11). From this previous study, it can be postulated that the blockade of NE toxicity may exert renoprotective effects. Sivelestat, a specific $\mathrm{NE}$ inhibitor, has been demonstrated to mitigate lung injury, such as pulmonary fibrosis (12) and acute lung injury (13). Of note, Suda et al found that sivelestat improved the survival of animals with sepsis (14). However, they only focused on the beneficial effects of sivelestat in attenuating lung damage. Apart from lung damage, sepsis often leads to impaired function in other vital organs, including the kidneys (15), liver (16) and heart (17). In order to fully evaluate the potential therapeutic effects of a drug or an agent in sepsis, assessing its effects on multiple organs is mandatory.

In the present study, we examined the effects of sivelestat on sepsis-associated AKI in a rat model of sepsis induced by cecal ligation and puncture (CLP) and also explored the underlying mechanisms. 


\section{Materials and methods}

Animals and ethics. Male Sprague-Dawley rats (weighing 200-250 g) were obtained from the Changsheng Biotech Co., Ltd. (Beijing, China) and housed under specific pathogen-free conditions at a constant temperature of $20-22^{\circ} \mathrm{C}$ and humidity of $50-60 \%$ with a 12 -h light/dark cycle, and were allowed free access to food and water. All animal experiments were performed in accordance with the Guidelines for the Care and Use of Laboratory Animals and were approved by the Institutional Animal Care and Use Committee of China Medical University, Shenyang, China.

CLP procedure and animal grouping. CLP procedure was performed to initiate sepsis in rats according to previously published protocols (18). In short, the rats were first anaesthetized by the intraperitoneal (i.p.) administration of $10 \%$ chloral hydrate (350 mg/kg; Sinopharm, Beijing, China), and a ventral midline incision $(1.5 \mathrm{~cm}$ in length) was made on the rats. The caecum was exposed, ligated, punctured with a gauge needle 3 times, and then placed back into the abdomen. Rats that underwent sham operation (the caecum was exposed by a ventral midline incision, but was not ligated or punctured) were used as the controls. Thereafter, the abdominal incision was closed in layers with 3-0 surgical sutures, and the rats were allowed to recover from the anaesthesia. These rats were randomly divided into 6 groups (n=8/group) as follows: i) group 1: the sham-operated group (Sham); ii) group 2: the sham-operated group administered the low dose of sivelestat (Sham $+\mathrm{L}$-sivelestat); iii) group 3: the sham-operated group administered the high dose of sivelestat (Sham + H-sivelestat); iv) group 4: the rats with sepsis who were not treated (Sepsis); v) group 5: the rats with sepsis who were administered the low dose of sivelestat (Sepsis + L-sivelestat); and vi) group 6: the rats with sepsis who were administered the high dose of sivelestat (Sepsis $+\mathrm{H}$-sivelestat). The rats in groups 2 and 3 , and 5 and 6 , rats were administered an i.p. injection of 50 or $100 \mathrm{mg} / \mathrm{kg}$ body weight sivelestat (Ono Pharmaceutical Co., Osaka, Japan) immediately after the sham-operation or the initiation of sepsis. The rats in groups 1 and 4 received an infusion of normal saline (vehicle) into the intraperitoneal cavity. The doses of sivelestat used in our study were selected based on our preliminary experiments (data not shown) and a previous study (12). Blood samples from each rat were obtained at 6 and 24 $\mathrm{h}$ post-surgery, and the rat kidneys were rapidly removed at $24 \mathrm{~h}$ post-surgery after sacrifice (by an overdose of anesthetics) and immediately frozen in liquid nitrogen or fixed in $10 \%$ formaldehyde (Sinopharm) for use in subsequent experiments.

In order to determine whether sivelestat affects animal survival, the mortalities were measured in another set of rats treated with or without sivelestat up to $108 \mathrm{~h}$ after the procedure ( $n=10 /$ group). In addition, the mean arterial pressure (MAP) in these rats was assessed using a non-invasive blood pressure monitoring system at $24 \mathrm{~h}$ according to the manufacturer's instructions (ALC-NIBP, Alcott Biotech Co., Ltd., Shanghai, China).

Measurement of serum biochemical parameters. Serum samples were centrifuged at 1,000 rpm for $20 \mathrm{~min}$, and then subjected to the detection of blood urea nitrogen (BUN), neutrophil gelatinase-associated lipocalin (NGAL) levels, tumor necrosis factor $\alpha$ (TNF- $\alpha$ ) and interleukin-1 $\beta$ (IL-1 $\beta$ ) levels and $\mathrm{NE}$ levels in the serum and renal tissues using commercially available kits (Boster, Uscn Life Science, Inc., Wuhan, China) according to the manufacturer's instructions.

Glomerular filtration rate (GFR) and fractional excretion of sodium $\left(F E_{N a}\right)$ assessments. The rats that underwent CLP or sham surgery were anaesthetized at $24 \mathrm{~h}$ and subjected to the following assessments. GFR was determined by measuring the inulin clearance. In brief, inulin (Sigma-Aldrich, St. Louis, MO, USA) was dissolved in normal saline to a concentration of $5 \mathrm{mg} / \mathrm{ml}$, and then administered through the femoral vein at a dose of $1 \mathrm{ml} / \mathrm{h} / 100 \mathrm{~g}$ body weight. Following equilibration, the urinary bladder was cannulated to collect a 30 -min urine sample. In addition, a blood sample $(1 \mathrm{ml})$ was collected at the midpoint of the urine collection period. Ten minutes later, urine and blood samples were collected again. Inulin concentrations in the urine and plasma were determined using the anthrone method, while sodium and creatinine concentrations were determined using commercial assay kits (Nanjing Jiancheng Bioengineering Institute, Nanjing, China), respectively. The values of GFR and $\mathrm{FE}_{\mathrm{Na}}$ were calculated through standard formulas, as previously described (19-21): GFR $=[$ (urine inulin) $\mathrm{x}$ (volume of urine collected)]/[(plasma inulin) $\mathrm{x}$ (time of collection)], and expressed as $\mathrm{ml} / \mathrm{min} / 100 \mathrm{~g}$ body weight; $\mathrm{FE}_{\mathrm{Na}}=100 \times[$ (urine sodium $\mathrm{x}$ plasma creatinine)/(plasma sodium $\mathrm{x}$ urine creatinine)].

Hematoxylin and eosin $(H \& E)$ staining and immunohistochemistry. The formaldehyde-fixed kidney tissues were embedded in paraffin, cut into 5- $\mu \mathrm{m}$-thick sections, and then deparaffinized in xylene and hydrated in graded ethanol. For the morphological examination, these slices were stained with hematoxylin (Solarbio, Beijing, China) and eosin (Sinopharm). For immunohistochemistry, these sections were heated in citrate buffer at $100^{\circ} \mathrm{C}$ for $10 \mathrm{~min}$ to retrieve the antigen and then treated with 3\% hydrogen peroxide (both from Sinopharm) at room temperature for 15 min to block the endogenous peroxidase activity. After being blocked with normal goat serum at room temperature for $15 \mathrm{~min}$, these sections were incubated with rabbit polyclonal antibodies against high-mobility group box 1 (HMGB1; 1:100 dilution, bs-0664R), inducible nitric oxide (NO) synthase (iNOS; 1:100 dilution, bs-0162R) (both from Bioss, Beijing, China) and a mouse monoclonal antibody against ED-1 (1:50 dilution, sc-59103; Santa Cruz Biotechnology, Santa Cruz, CA, USA) at $4^{\circ} \mathrm{C}$ overnight. These sections were then incubated with biotin-labeled goat anti-rabbit IgG (1:200 dilution, A0277) or anti-mouse $\operatorname{IgG}(1: 200$ dilution, $\mathrm{A} 0286)$ at $37^{\circ} \mathrm{C}$ for $30 \mathrm{~min}$, and treated with horseradish peroxidase (HRP)-conjugated streptavidin (all from Beyotime, Shanghai, China) for an additional $30 \mathrm{~min}$. Finally, the tissue sections visualized with 3,3'-diaminobenzidine (DAB) and counterstained with hematoxylin (both from Solarbio), and evaluated under a light microscope (Olympus, Tokyo, Japan).

Western blot analysis. Total protein samples were extracted from the kidney tissues using RIPA buffer (Beyotime) and the protein concentrations were evaluated by bicinchoninic acid assay. An equivalent amount of each protein sample ( $40 \mu \mathrm{g})$ 
was loaded and separated through sodium dodecyl sulphate (SDS)-polyacrylamide gel electrophoresis (PAGE) and transferred onto polyvinylidene difluoride membranes (PVDF; Millipore, Bedford, MA, USA). The membranes were then blocked with $5 \%$ non-fat milk, and incubated overnight at $4^{\circ} \mathrm{C}$ with rabbit polyclonal antibodies against NE (1:500 dilution, bs-6982R), HMGB1 (1:500 dilution, bs-0664R), iNOS (1:500 dilution, bs-0162R), serine/threonine kinase (Akt; 1:500 dilution, bs-6951R) and phosphorylated Akt (p-Akt; 1:500 dilution, bs-0876R). All primary antibodies were purchased from Bioss. Thereafter, the membranes were incubated with HRP-conjugated secondary antibodies (1:5000 dilution, A0208; Beyotime) at $37^{\circ} \mathrm{C}$ for $45 \mathrm{~min}$ and visualized using an enhanced chemiluminescence (ECL) system (Seven Sea Pharmtech Co., Ltd., Shanghai, China). The protein expression levels were expressed as a ratio to the endogenous control, $\beta$-actin.

Statistical analysis. All data are presented as the means \pm standard deviation (SD), and analyzed using GraphPad Prism version 6.0 or SPSS version 20.0. One-way analysis of variance (ANOVA) was performed on data obtained from the same time point, whilst two-way ANOVA was performed on data from different time points. The Bonferroni test was utilized for post hoc comparisons between different groups. A P-value $<0.05$ was considered to indicate a statistically significant difference. Survival data were analyzed by the Kaplan-Meier curve and log-rank (Mantel-Cox) test.

\section{Results}

Sivelestat decreases NE expression in rats with sepsis. We first evaluated whether the NE levels were affected by the CLP procedure using an ELISA kit and western blot analysis. Our results revealed that the administration of sivelestat alone generated no changes in comparison with the sham-operated rats (Fig. 1). The serum NE level was significantly increased at both 6 and 24 h post-CLP surgery, whereas decreased after sivelestat treatment (Fig. 1A). In addition, renal NE expression was significantly enhanced at $24 \mathrm{~h}$ post-CLP surgery, whereas it was suppressed by treatment with sivelestat (Fig. 1B and C). The higher dose of sivelestat was more effective than the lower dose.

Sivelestat promotes the survival and preserves the kidney function of rats with sepsis. Another set of rats (10 per group) was subjected to survival analysis for $108 \mathrm{~h}$. As indicated in Fig. 2A, no sham-operated rats treated with 50 or $100 \mathrm{mg} /$ $\mathrm{kg}$ sivelestat or the vehicle died during the whole experimental period. All rats in the sepsis group were dead at $108 \mathrm{~h}$ postCLP surgery, while 4 and 6 septic rats survived for $108 \mathrm{~h}$ when treated with $50(\mathrm{P}=0.062$ vs. sepsis group) and $100 \mathrm{mg} /$ $\mathrm{kg}$ sivelestat ( $\mathrm{P}<0.01$ vs. sepsis group), respectively (Fig. $2 \mathrm{~A})$. As compared with the sham-operated group $(104 \pm 5.8 \mathrm{mmHg})$, CLP induced a reduction in MAP $(89 \pm 7.2 \mathrm{mmHg})(\mathrm{P}<0.001)$, which was restored by sivelestat (Fig. 2B). Morphological changes in the rat kidneys were determined by H\&E staining and the parameters related to renal function were detected using commercial kits. Pathological alterations in the rat kidneys were characterized by the loss of the brush border, tubular cell
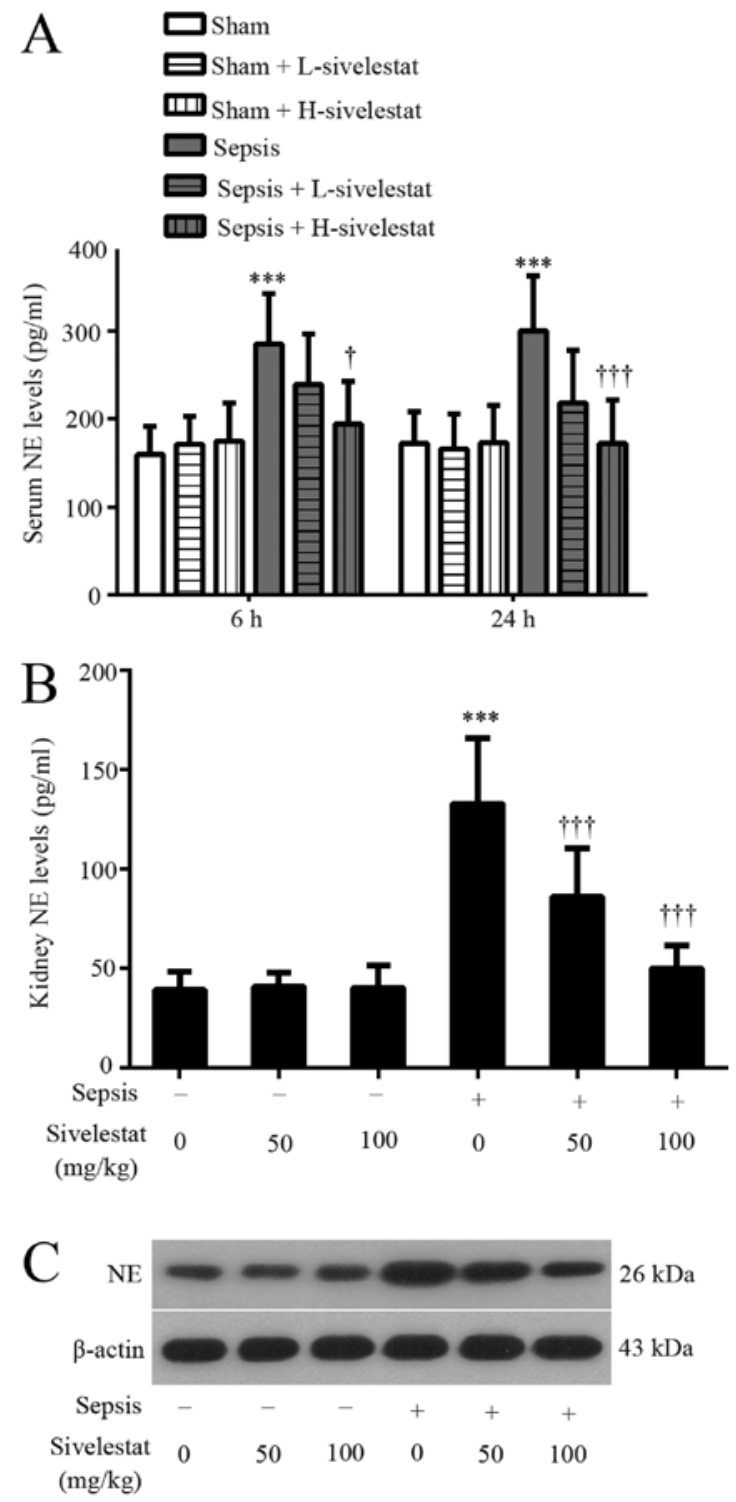

Figure 1. Sivelestat suppresses serum and kidney neutrophil elastase (NE) expression in septic rats. NE levels in (A) rat serum at 6 and $24 \mathrm{~h}$ and (B) kidney tissues at $24 \mathrm{~h}$ post-cecal ligation and puncture (CLP) surgery assessed using an ELISA kit ( $\mathrm{n}=8$ rats per group). (C) NE protein expression was detected by western blot analysis in rat kidney tissues at $24 \mathrm{~h}$ post-CLP procedure $(n=5)$. $\beta$-actin was used as an endogenous control. Data are expressed as the means \pm SD. No significant differences were observed in the sham-operated rats. ${ }^{* * *} \mathrm{P}<0.001$ compared with the sham-operated (Sham) group; ${ }^{\dagger} \mathrm{P}<0.05$, ${ }^{+\dagger} \mathrm{P}<0.001$ compared with the sepsis group at the same time point.

flattening and tubular lumen dilation, while the administration of sivelestat partly reversed these changes (Fig. 2C). In addition, sivelestat did not affect the serum BUN levels, NGAL levels, inulin clearance and $\mathrm{FE}_{\mathrm{Na}}$ in the rats that underwent sham surgery (P>0.05; Fig. 2D-G). However, CLP-induced a significant upregulation in serum BUN and NGAL levels in the rats and this effect was suppressed by sivelestat, with the higher dose being more effective (Fig. 2D and E). Additionally, the decreased inulin clearance (indicating reduced GFR) in the rats with sepsis was partly reversed by sivelestat (Fig. 2F). The CLP-induced increase in $\mathrm{FE}_{\mathrm{Na}}$ (indicating tubular dysfunction) was slightly reduced by sivelestat, but failed to reach statistical significance (Fig. 2G). Collectively, the administration of 
A

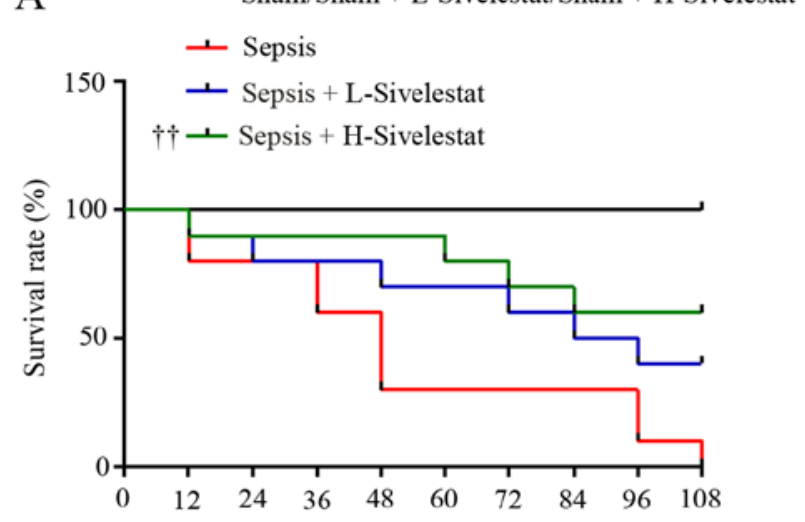

B

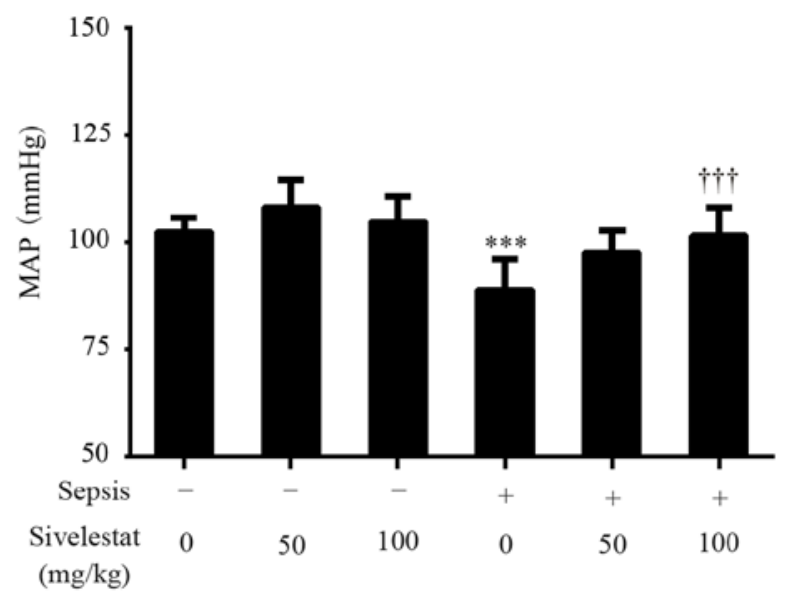

$\mathrm{C}$
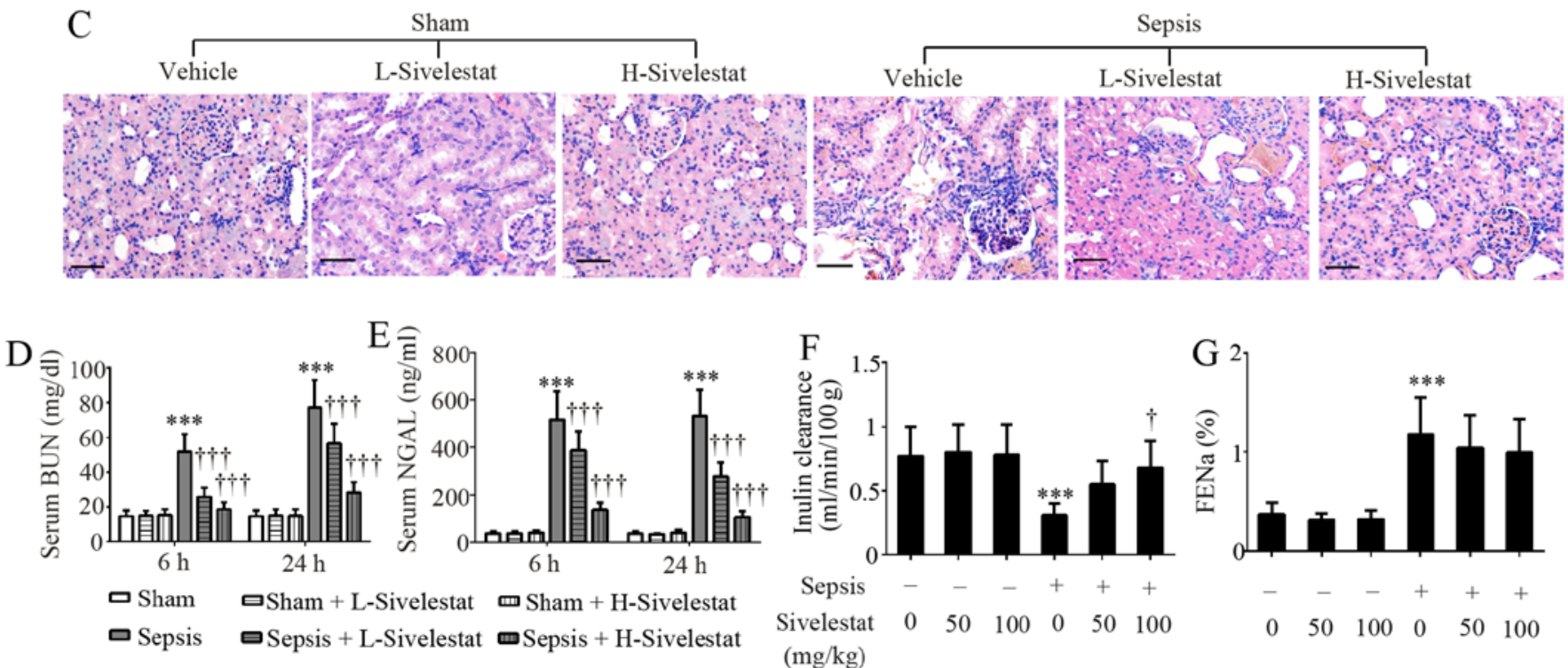

Figure 2. Effects of sivelestat on kidney function in rats that underwent sham or cecal ligation and puncture (CLP) surgery. (A) Kaplan-Meier survival plots for all experimental rats ( $\mathrm{n}=10$ rats per group). ${ }^{\dagger} \mathrm{P}<0.01$, compared with the sepsis group (log-rank test). (B) Rat mean arterial pressure (MAP) was detected at $24 \mathrm{~h}$ post-surgery ( $\mathrm{n}=8-10$ rats per group). (C) Representatives of hematoxylin and eosin (H\&E) staining of rat kidney tissues. (D) Serum levels of blood urea nitrogen (BUN), (E) neutrophil gelatinase-associated lipocalin (NGAL) were determined at 6 and $24 \mathrm{~h}$ post-surgery using commercially available kits (n=8 rats per group). ( $\mathrm{F})$ Inulin clearance and $(\mathrm{G}) \mathrm{FE}_{\mathrm{Na}}$ were determined at $24 \mathrm{~h}$ post-surgery $\left(\mathrm{n}=8\right.$ rats per group). Data are expressed as the means $\pm \mathrm{SD}$. ${ }^{* * *} \mathrm{P}<0.001$ compared with the sham-operated (Sham) group; ${ }^{\dagger} \mathrm{P}<0.05,{ }^{\dagger} \mathrm{P}<0.01,{ }^{1 \dagger} \mathrm{P}<0.001$ compared with the sepsis group at the same time point. Scale bars, $50 \mu \mathrm{m}$.

sivelestat protected the rats against sepsis induced by multiple bacterial infection. Since the lower dose of sivelestat did not induce mortality, kidney distortion or renal dysfunction in the sham-operated rats, this group was excluded from the following mechanism experiments.

Sivelestat suppresses the release of pro-inflammatory mediators and macrophage infiltration induced by CLP in rats. The overproduction of pro-inflammatory mediators in response to bacterial infection is one of the major characteristics of sepsis (22). Therefore, the serum levels of TNF- $\alpha$ and IL- $1 \beta$ were assessed in this study. We found that the levels of these two pro-inflammatory cytokines were significantly increased at 6 and $24 \mathrm{~h}$ post-CLP procedure (Fig. 3A). Although sivelestat alone generated no changes in the serum levels of TNF- $\alpha$ and IL-1 $\beta$ as compared to the sham-operated group $(\mathrm{P}>0.05)$, a marked inhibitory effect of sivelestat on these two pro-inflammatory cytokines in the rats with sepsis rats was observed (Fig. 3A). Immunohistochemistry was also performed in the renal tissues to detect the ED-1-positive macrophages (a marker of macrophage infiltration) at $24 \mathrm{~h}$ after the surgical procedures (Fig. 3B). As indicated in Fig. 3C, the number of renal ED-1-positive cells was increased from $1.63 \pm 1.92$ to $13.38 \pm 5.29$ per $0.095 \mathrm{~mm}^{2}$ after CLP surgery, but was decreased by the administration of sivelestat $(9.13 \pm 2.64$ by L-sivelestat; $5.38 \pm 1.77$ by H-sivelestat). Unlike TNF- $\alpha$ and IL-1 $\beta$ that are released immediately in response to bacterial infection, HMGB1 is a late pro-inflammatory mediator (23). We therefore wished to determine whether sivelestat can reduce HMGB1 expression by detecting its renal expression at $24 \mathrm{~h}$ after the sham or CLP procedure using immunohistochemical staining and western blot analysis. A weak nuclear expression of HMGB1 was observed in a few renal parenchymal cells in the control kidney tissues (Fig. 3B). However, the number of HMGB1-positive cells was significantly increased after the CLP procedure, whereas it was decreased by treatment 
A
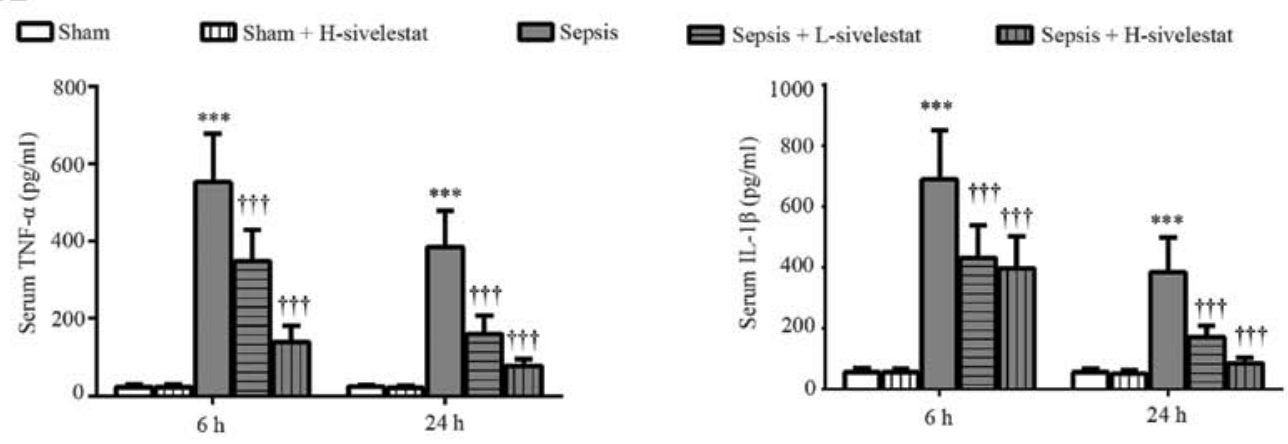

B

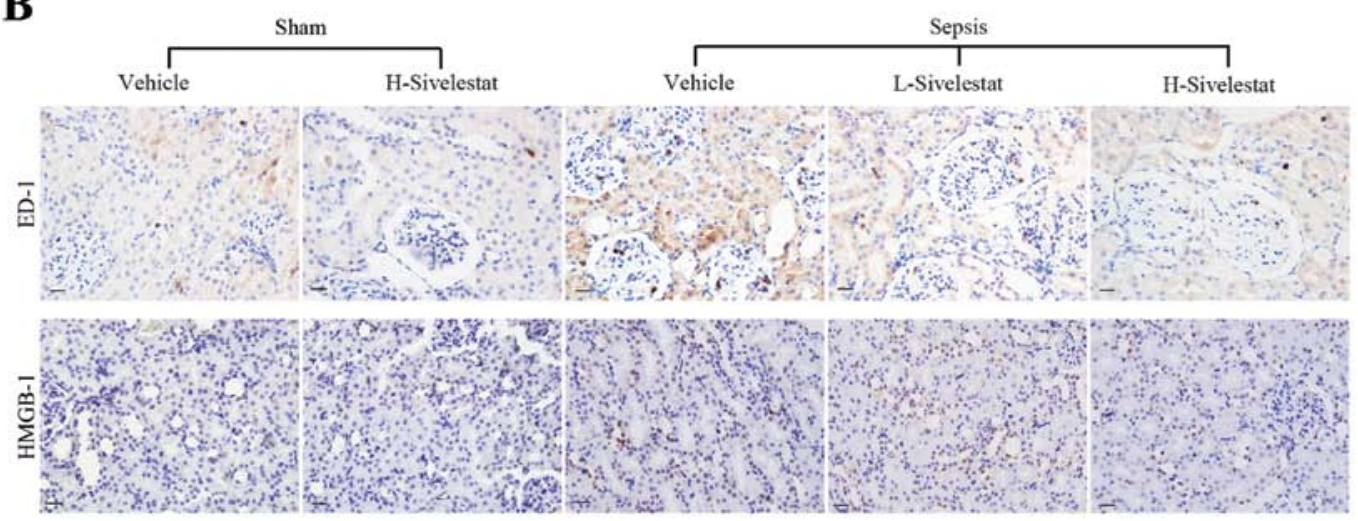

C

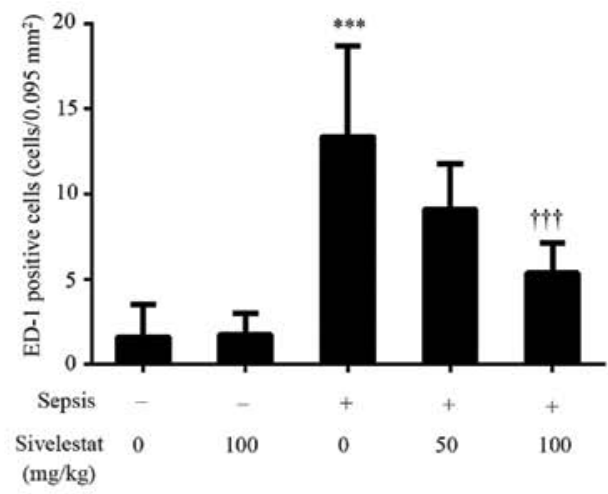

D

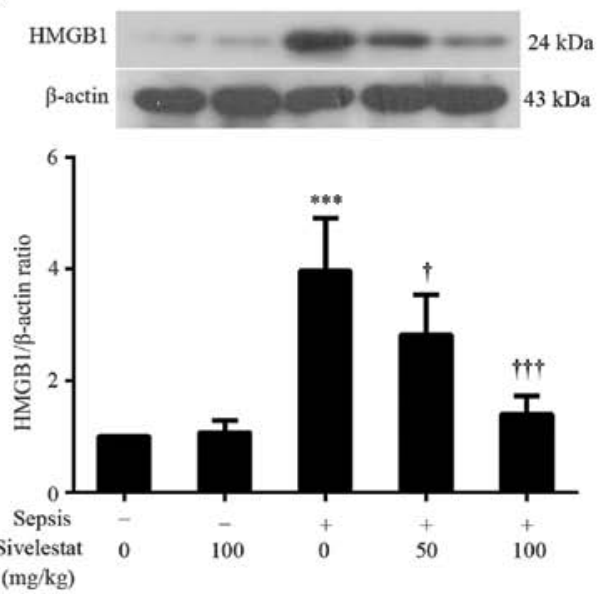

Figure 3. Sivelestat inhibits cecal ligation and puncture (CLP)-induced macrophage infiltration and pro-inflammation mediator release in rats. (A) Serum concentrations of tumor necrosis factor $\alpha$ (TNF- $\alpha$ ) and interleukin-1 $\beta$ (IL-1 $\beta$ ) were determined using the respective ELISA kits at 6 and $24 \mathrm{~h}$ after sham or CLP surgery ( $\mathrm{n}=8$ rats per group). (B) Macrophage infiltration in rat kidney tissues was assessed with specific antibody against ED-1 at $24 \mathrm{~h}$ after sham or CLP surgery (upper panels), and (C) the ED-1 positive cells were counted under a light microscope. (B) Representative images of immunohistochemical staining for high-mobility group box 1 (HMGB1) in rat kidney tissues at $24 \mathrm{~h}$ post-surgery (lower panels). (D) Renal HMGB1 protein expression was detected by western blot analysis $\left(\mathrm{n}=5\right.$ ). $\beta$-actin was used as an endogenous control. Data are expressed as the means $\pm \mathrm{SD} .{ }^{* * *} \mathrm{P}<0.001$ compared with the sham-operated (Sham) group; ${ }^{\dagger} \mathrm{P}<0.05,{ }^{\dagger} \mathrm{P}<0.001$ compared with the sepsis group at the same time point. Scale bars, $20 \mu \mathrm{m}$.

with sivelestat at different doses (Fig. 3B). The results from western blot analysis confirmed the immunohistochemical results (Fig. 3D). The above-mentioned results indicate an antiinflammatory effect of sivelestat in a model of sepsis-related AKI.

Sivelestat inhibits renal iNOS expression in septic rats. The polymicrobial infection induced-overproduction of pro-inflammatory mediators can accelerate the release of powerful secondary mediators, such as NO (24). Given the fact that the production of NO is predominantly mediated by iNOS (25), we evaluated renal iNOS protein expression by using immunohistochemical staining and western blot analysis. We found that iNOS was expressed in the cytoplasm of renal tubule epithelial cells (Fig. 4A). As compared with the normal kidney tissues, the more intense expression of iNOS was observed in the septic kidney tissues (Fig. 4A). However, the upregulation of iNOS expression was significantly inhibited by treatment with sivelestat (Fig. 4A). These results were confirmed by western blot analysis (Fig. 4B). 


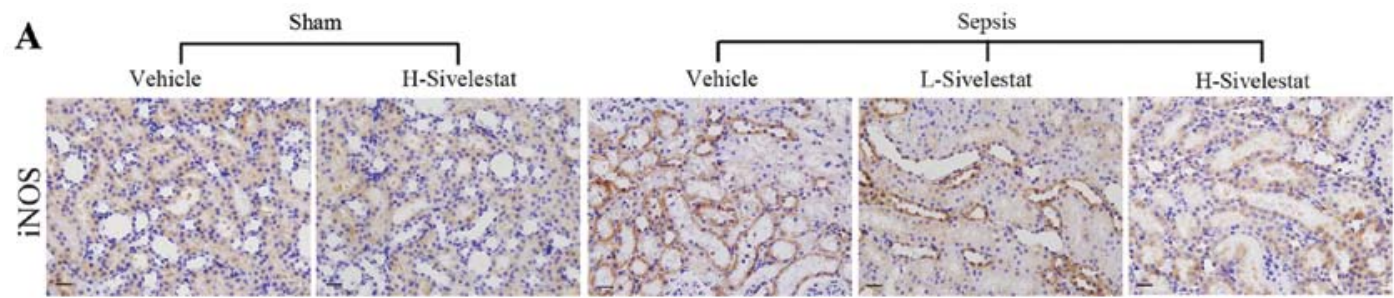

B
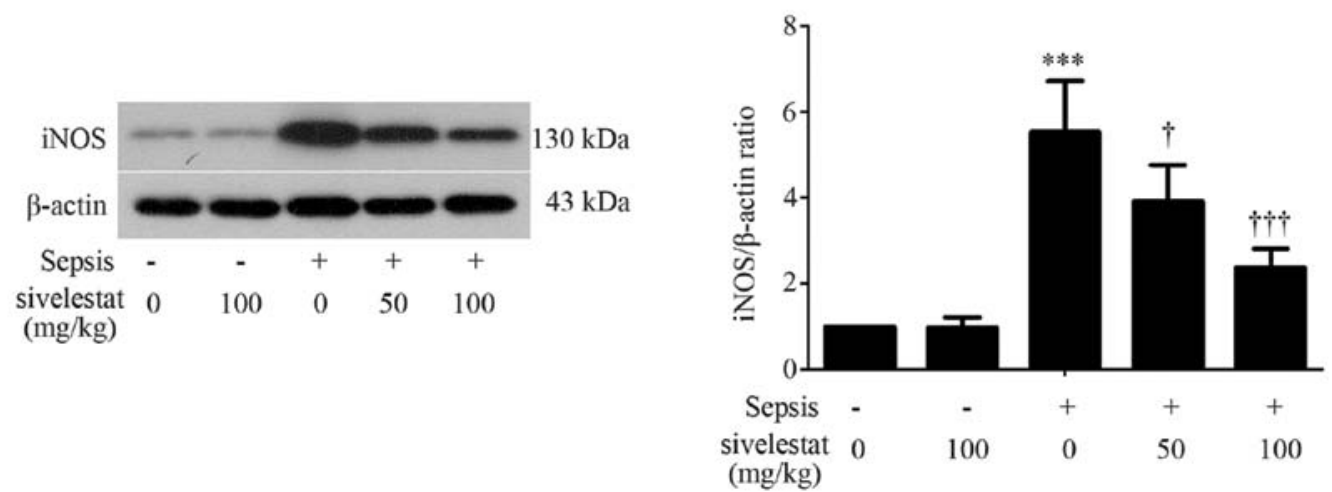

Figure 4. Sivelestat inhibits cecal ligation and puncture (CLP)-induced nitric oxide synthase (iNOS) upregulation in rat kidney tissues. (A) Representative images of immunohistochemical staining fir iNOS in rat kidney tissues at $24 \mathrm{~h}$ post-CLP procedure. (B) Renal iNOS protein expression was detected by western blot analysis $(n=5)$. $\beta$-actin was used as an endogenous control. Data are expressed as the means $\pm S D$. ${ }^{* * *} \mathrm{P}<0.001$ compared with the sham-operated (Sham) group; ${ }^{\dagger} \mathrm{P}<0.05,{ }^{\dagger \dagger} \mathrm{P}<0.001$ compared with the sepsis group. Scale bars, $20 \mu \mathrm{m}$.

A

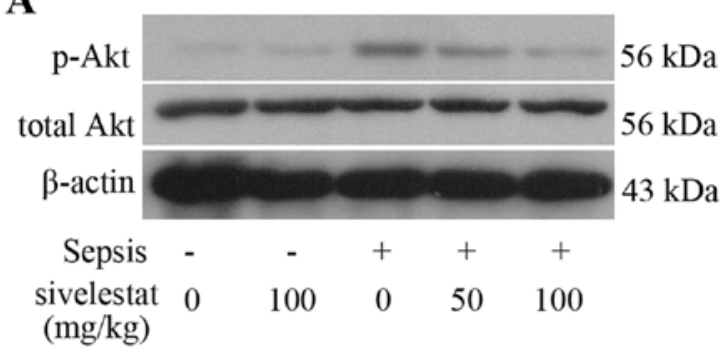

B

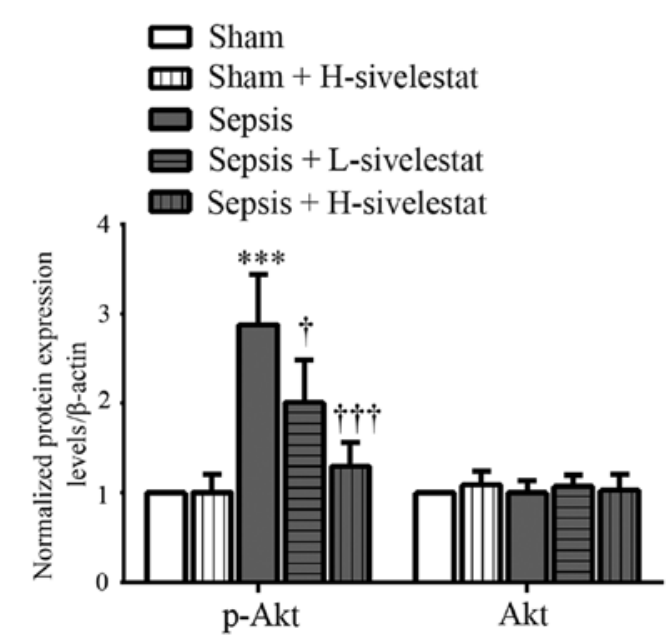

Figure 5. Sivelestat inhibits the cecal ligation and puncture (CLP)-induced activation of the phosphatidylinositol 3-kinase (PI3K)/Akt pathway activation in rat kidney tissues. (A) Representative blots for total Akt and phospho-Akt (p-Akt) in rat kidney tissues at $24 \mathrm{~h}$ post-CLP procedure $(\mathrm{n}=5)$. $\beta$-actin was used as an endogenous control. (B) Protein expression levels of total Akt and p-Akt were normalized as ratios to $\beta$-actin. Data are expressed as the means $\pm \mathrm{SD} .{ }^{* * *} \mathrm{P}<0.001$ compared with the sham-operated (Sham) group; ${ }^{\dagger} \mathrm{P}<0.05,{ }^{\dagger \dagger} \mathrm{P}<0.001$ compared with the sepsis group.
Sivelestat inhibits the activation of the Akt signaling pathway in the kidney tissues of rats with sepsis. The activation of the Akt pathway was assessed by western blot analysis of the Akt phosphorylation product, p-Akt in the rat kidney tissues at $24 \mathrm{~h}$ post-surgery. We noted that total Akt protein expression in the kidney tissues remained unaltered in the different experimental groups (Fig. 5). Treatment with sivelestat alone had no significant effect on renal p-Akt expression in the rats subjected to sham operation (Fig. 5). However, a marked increase in the phosphorylation levels of Akt was observed in the septic renal tissues. The high phosphorylation levels of Akt induced by sepsis were suppressed after low-dose sivelestat treatment and the levels almost returned to normal after high-dose sivelestat treatment (Fig. 5). Our data thus suggest that the administration of sivelestat suppresses the CLP-induced activation of Akt in rat kidneys.

\section{Discussion}

Despite improvements in the supportive care of patients with sepsis, current therapeutic approaches are relatively ineffective due to the protean nature of septic $\operatorname{AKI}(3,26)$. A variety of animal models have been established to investigate the specific molecular events occurring in sepsis (27). The most frequently used model is the rodent model of CLP, in which sepsis originates from a polymicrobial infection $(18,28)$. While Hirche et al found that the absence of NE increased the mortality of mice to Pseudomonas aeruginosa infection (29), Suda et al found that the inhibition of NE improved the survival rate of rats that uderwent CLP (14). These earlier findings suggest that NE mediates innate host protection against bacterial infection; however, its on-going and excessive activation may have adverse effects. Our present study showed that sivelestat improved the survival of rats 
with sepsis and preserved their kidney functions, revealing the therapeutic role of sivelestat in sepsis-associated renal injury.

Sivelestat is a specific NE inhibitor first synthesized by Kawabata et al in 1991 (30), and has been reported to attenuate pulmonary inflammation and fibrosis in animal models $(12,31)$. Its effects on lung and cardiovascular diseases have been examined in several clinical trials (32-34). Of note, apart from the therapeutic effects of sivelestat in the lungs, it has also been demonstrated to have potential to reduce inflammation-related lesions in the liver (35) and pancreas (36) in preclinical disease models. Our study demonstrated that treatment with sivelestat alleviated the dysregulation in BUN and NGAL levels in the rats with sepsis. The CLP surgery-induced decrease in GFR and tubular function were partly restored by sivelestat, indicating a protective role of this NE inhibitor in sepsis-related kidney injury.

We then focused on the anti-inflammatory effects of sivelestat in septic AKI. Ischemia/reperfusion injury, bacterial infection or the nephrotoxic agent-induced aberrant infiltration of immune cells, such as neutrophils, macrophages and lymphocytes are considered to contribute to the pathogenesis of AKI (37). Pro-inflammatory mediators, such as TNF- $\alpha$ and IL-1 $\beta$ are released early in response to bacterial infection and can be acutely toxic (38). Therapeutic agents being able to reduce the release of TNF- $\alpha$ and/or IL-1 $\beta$ in murine septic models are suggested to ameliorate sepsis (39-41). The study by Suda et al revealed that sivelestat suppressed the aberrant release of TNF- $\alpha$ (not statistically significant) and IL-1 $\beta$ (statistically significant) in septic lungs (14). In this study, we found that abnormal macrophage infiltration and $\mathrm{TNF}-\alpha / \mathrm{IL}-1 \beta$ release were reduced by sivelestat. In contrast to other sepsis-associated cytokines, HMGB1 is a late pro-inflammatory cytokine released from monocytes and/or apoptotic cells after the onset of sepsis that further amplifies the inflammatory process (42). The neutralization of HMGB1 can therapeutically reverse lethality in experimental sepsis $(38,43)$. In this study, we demonstrated that the administration of sivelestat markedly reduced the CLP-induced upregulation of renal HMGB1. Such results were supported by an earlier study showing that sivelestat treatment reduced LPS-induced pulmonary HMGB1 upregulation in rats (44). Of note, most clinical trials targeting TNF (45) or IL-1 (46) in sepsis have failed. Therefore, further clinical studies examining the anti-inflammatory effects of sivelestat in sepsis are urgently required.

The polymicrobial infection-induced overproduction of pro-inflammatory mediators can accelerate the release of powerful secondary mediators, such as reactive nitrogen/oxygen species (47). The activation of iNOS during sepsis results in increased NO levels that causes tubular injury through the local generation of reactive nitrogen species, and the selective inhibition of iNOS has been suggested as a potential novel treatment for sepsis-induced AKI (24). In this study, we found that sivelestat was able to reduce CLP-induced renal iNOS overexpression in rats. Although previous studies have indicated an inhibitory effect of sivelestat on iNOS $(48,49)$, as far as we know, our study was the first to show such an effect in septic kidneys.
Previous studies searching for novel anti-inflammatory agents have suggested a critical role of phosphatidylinositol 3-kinase (PI3K)/Akt signaling in sepsis. It is worth noting that the phosphorylation of Akt referring to PI3K/Akt pathway activation is enhanced in the lungs (50) and liver (51), but is weakened in the heart (52) after the CLP procedure or LPS challenge. Consequently, both the blockade and activation of PI3K/Akt signaling transduction have been shown to improve outcome in septic shock. Of note, a previous study by Sadhu et al showed that the inactivation of the PI3K/Akt pathway with a pharmaceutical inhibitor blocked TNF1 $\alpha$ stimulated NE exocytosis (53). Since sivelestat can suppress IL-1 $\beta$-stimulated Akt phosphorylation in hepatocytes (54), we explored whether sivelestat also has an inhibitory effect on PI3K/Akt signaling transduction in septic kidneys in this study. We found that CLP-enhanced renal Akt phosphorylation was decreased by sivelestat. Moreover, the LPS induction of the phosphorylation of Akt is responsible for nuclear factor $-\kappa \mathrm{B}(\mathrm{NF}-\kappa \mathrm{B})$ activation in human renal mesangial cells (55), and the inhibitory effects of sivelestat on $N F-\kappa B$ signals have been previously reported (36). It is likely that Akt signals are involved in the regulatory effects of sivelestat on the NF- $\mathrm{NB}$ pathway. Further in vitro experiments are thus being carried out by our group to study the underlying mechanisms.

In conclusion, in this study, we demonstrate that the administration of the NE inhibitor, sivelestat, mitigates CLP-induced kidney injury, reduces inflammation, and suppresses the activation of the Akt signaling pathway. Our study suggests that sivelestat has potential to attenuate sepsis-induced kidney injury.

\section{Acknowledgements}

This study was supported by grants from the National Natural Science Foundation of China (no. 81471847), the Natural Science Foundation of Liaoning Province (no. 2014021003), and the Science and Technology Project of Shenyang City (no. F14-158-9-40).

\section{References}

1. Fry DE: Sepsis, systemic inflammatory response, and multiple organ dysfunction: the mystery continues. Am Surg 78: 1-8, 2012.

2. Rittirsch D, Flierl MA and Ward PA: Harmful molecular mechanisms in sepsis. Nat Rev Immunol 8: 776-787, 2008.

3. Morrell ED, Kellum JA, Pastor-Soler NM and Hallows KR: Septic acute kidney injury: molecular mechanisms and the importance of stratification and targeting therapy. Crit Care 18: 501,2014

4. Chen L, Yang S, Zumbrun EE, Guan H, Nagarkatti PS and Nagarkatti M: Resveratrol attenuates lipopolysaccharide-induced acute kidney injury by suppressing inflammation driven by macrophages. Mol Nutr Food Res 59: 853-864, 2015.

5. Shimazu R, Akashi S, Ogata H, Nagai Y, Fukudome K, Miyake K and Kimoto M: MD-2, a molecule that confers lipopolysaccharide responsiveness on toll-like receptor 4. J Exp Med 189: 1777-1782, 1999.

6. Villar J, Cabrera N, Casula M, Flores C, Valladares F, Muros M, Blanch L, Slutsky AS and Kacmarek RM: Mechanical ventilation modulates toll-like receptor signaling pathway in a sepsis-induced lung injury model. Intensive Care Med 36: 1049-1057, 2010.

7. Bang BR, Kim SJ, Yagita H, Croft $M$ and Kang YJ: Inhibition of 4-1BBL-regulated TLR response in macrophages ameliorates endotoxin-induced sepsis in mice. Eur J Immunol 45: 886-892, 2015. 
8. Opal SM, Laterre PF, Francois B, LaRosa SP, Angus DC, Mira JP, Wittebole X, Dugernier T, Perrotin D, Tidswell M, et al; ACCESS Study Group: Effect of eritoran, an antagonist of MD2-TLR4, on mortality in patients with severe sepsis: the ACCESS randomized trial. JAMA 309: 1154-1162, 2013.

9. Griffin KL, Fischer BM, Kummarapurugu AB, Zheng S, Kennedy TP, Rao NV, Foster WM and Voynow JA: 2-O, 3-O-desulfated heparin inhibits neutrophil elastase-induced HMGB-1 secretion and airway inflammation. Am J Respir Cell Mol Biol 50: 684-689, 2014.

10. Korkmaz B, Horwitz MS, Jenne DE and Gauthier F: Neutrophil elastase, proteinase 3 , and cathepsin $\mathrm{G}$ as therapeutic targets in human diseases. Pharmacol Rev 62: 726-759, 2010.

11. Zager RA, Johnson AC and Frostad KB: Rapid renal alpha-1 antitrypsin gene induction in experimental and clinical acute kidney injury. PLoS One 9: e98380, 2014

12. Takemasa A, Ishii Y and Fukuda T: A neutrophil elastase inhibitor prevents bleomycin-induced pulmonary fibrosis in mice. Eur Respir J 40: 1475-1482, 2012.

13. Ishii T, Doi K, Okamoto K, Imamura M, Dohi M, Yamamoto K, Fujita T and Noiri E: Neutrophil elastase contributes to acute lung injury induced by bilateral nephrectomy. Am J Pathol 177: $1665-1673,2010$

14. Suda K, Takeuchi H, Hagiwara T, Miyasho T, Okamoto M, Kawasako K, Yamada S, Suganuma K, Wada N, Saikawa Y, et al: Neutrophil elastase inhibitor improves survival of rats with clinically relevant sepsis. Shock 33: 526-531, 2010.

15. Schortgen F and Asfar P: Update in sepsis and acute kidney injury 2014. Am J Respir Crit Care Med 191: 1226-1231, 2015.

16. Yan J, Li S and Li S: The role of the liver in sepsis. Int Rev Immunol 33: 498-510, 2014.

17. Drosatos K, Lymperopoulos A, Kennel PJ, Pollak N, Schulze PC and Goldberg IJ: Pathophysiology of sepsis-related cardiac dysfunction: driven by inflammation, energy mismanagement, or both? Curr Heart Fail Rep 12: 130-140, 2015.

18. Rittirsch D, Huber-Lang MS, Flierl MA and Ward PA: Immunodesign of experimental sepsis by cecal ligation and puncture. Nat Protoc 4: 31-36, 2009.

19. Schick MA, Baar W, Flemming S, Schlegel N, Wollborn J, Held C, Schneider R, Brock RW, Roewer N and Wunder C: Sepsis-induced acute kidney injury by standardized colon ascendens stent peritonitis in rats - a simple, reproducible animal model. Intensive Care Med Exp 2: 34, 2014.

20. Souza AC, Volpini RA, Shimizu MH, Sanches TR, Camara NO, Semedo P, Rodrigues CE, Seguro AC and Andrade L: Erythropoietin prevents sepsis-related acute kidney injury in rats by inhibiting NF- $\kappa \mathrm{B}$ and upregulating endothelial nitric oxide synthase. Am J Physiol Renal Physiol 302: F1045-F1054, 2012.

21. Kadova Z, Dolezelova E, Cermanova J, Hroch M, Laho T, Muchova L, Staud F, Vitek L, Mokry J, Chladek J, et al: IL-1 receptor blockade alleviates endotoxin-mediated impairment of renal drug excretory functions in rats. Am J Physiol Renal Physiol 308: F388-F399, 2015.

22. King EG, Bauzá GJ, Mella JR and Remick DG: Pathophysiologic mechanisms in septic shock. Lab Invest 94: 4-12, 2014.

23. Diener KR, Al-Dasooqi N, Lousberg EL and Hayball JD: The multifunctional alarmin HMGB1 with roles in the pathophysiology of sepsis and cancer. Immunol Cell Biol 91: 443-450, 2013.

24. Heemskerk S, Masereeuw R, Russel FG and Pickkers P: Selective iNOS inhibition for the treatment of sepsis-induced acute kidney injury. Nat Rev Nephrol 5: 629-640, 2009.

25. Lowenstein CJ and Padalko E: iNOS (NOS2) at a glance. J Cell Sci 117: 2865-2867, 2004

26. Fink MP and Warren HS: Strategies to improve drug development for sepsis. Nat Rev Drug Discov 13: 741-758, 2014.

27. Buras JA, Holzmann B and Sitkovsky M: Animal models of sepsis: setting the stage. Nat Rev Drug Discov 4: 854-865, 2005.

28. Brooks HF, Osabutey CK, Moss RF, Andrews PL and Davies DC: Caecal ligation and puncture in the rat mimics the pathophysiological changes in human sepsis and causes multi-organ dysfunction. Metab Brain Dis 22: 353-373, 2007.

29. Hirche TO, Benabid R, Deslee G, Gangloff S, Achilefu S, Guenounou M, Lebargy F, Hancock RE and Belaaouaj A: Neutrophil elastase mediates innate host protection against Pseudomonas aeruginosa. J Immunol 181: 4945-4954, 2008.

30. Kawabata K, Suzuki M, Sugitani M, Imaki K, Toda M and Miyamoto T: ONO-5046, a novel inhibitor of human neutrophil elastase. Biochem Biophys Res Commun 177: 814-820, 1991.
31. Yoshikawa N, Inomata T, Okada Y, Shimbo T, Takahashi M, Akita K, Uesugi Y and Narumi Y: Sivelestat sodium hydrate reduces radiation-induced lung injury in mice by inhibiting neutrophil elastase. Mol Med Rep 7: 1091-1095, 2013.

32. Kohira S, Oka N, Inoue N, Itatani K, Kitamura T, Horai T, Oshima H, Tojo K, Yoshitake S and Miyaji K: Effect of additional preoperative administration of the neutrophil elastase inhibitor sivelestat on perioperative inflammatory response after pediatric heart surgery with cardiopulmonary bypass. Artif Organs 38: 1018-1023, 2014.

33. Nomura N, Asano M, Saito T, Nakayama T and Mishima A: Sivelestat attenuates lung injury in surgery for congenital heart disease with pulmonary hypertension. Ann Thorac Surg 96: 2184-2191, 2013

34. Tagami T, Tosa R, Omura M, Fukushima H, Kaneko T, Endo T, Rinka H, Murai A, Yamaguchi J, Yoshikawa K, et al: Effect of a selective neutrophil elastase inhibitor on mortality and ventilatorfree days in patients with increased extravascular lung water: a post hoc analysis of the PiCCO Pulmonary Edema Study. J Intensive Care 2: 67, 2014.

35. Sakai S, Tajima H, Miyashita T, Nakanuma S, Makino I, Hayashi H, Nakagawara H, Kitagawa H, Fushida S, Fujimura T, et al: Sivelestat sodium hydrate inhibits neutrophil migration to the vessel wall and suppresses hepatic ischemia-reperfusion injury. Dig Dis Sci 59: 787-794, 2014.

36. Cao J and Liu Q: Protective effects of sivelestat in a caerulein-induced rat acute pancreatitis model. Inflammation 36: $1348-1356,2013$

37. Jang HR and Rabb H: Immune cells in experimental acute kidney injury. Nat Rev Nephrol 11: 88-101, 2015.

38. Yang H, Ochani M, Li J, Qiang X, Tanovic M, Harris HE, Susarla SM, Ulloa L, Wang H, DiRaimo R, et al: Reversing established sepsis with antagonists of endogenous high-mobility group box 1. Proc Natl Acad Sci USA 101: 296-301, 2004.

39. Lingaraju MC, Pathak NN, Begum J, Balaganur V, Ramachandra HD, Bhat RA, Ram M, Singh V, Kandasamy K, Kumar D, et al: Betulinic acid attenuates renal oxidative stress and inflammation in experimental model of murine polymicrobial sepsis. Eur J Pharm Sci 70: 12-21, 2015.

40. Carrino DA, Lidor C, Edelstein S and Caplan AI: Proteoglycan synthesis in vitamin D-deficient cartilage: recovery from vitamin D deficiency. Connect Tissue Res 19: 135-147, 1989.

41. Zhang H, Wang W, Fang H, Yang Y, Li X, He J, Jiang X, Wang W, Liu S, Hu J, et al: GSK-3 $\beta$ inhibition attenuates CLP-induced liver injury by reducing inflammation and hepatic cell apoptosis. Mediators Inflamm 2014: 629507, 2014.

42. Scaffidi P, Misteli T and Bianchi ME: Release of chromatin protein HMGB1 by necrotic cells triggers inflammation. Nature 418: 191-195, 2002.

43. Wang H, Liao H, Ochani M, Justiniani M, Lin X, Yang L, Al-Abed Y, Wang H, Metz C, Miller EJ, et al: Cholinergic agonists inhibit HMGB1 release and improve survival in experimental sepsis. Nat Med 10: 1216-1221, 2004.

44. Hagiwara S, Iwasaka H, Togo K and Noguchi T: A neutrophil elastase inhibitor, sivelestat, reduces lung injury following endotoxin-induced shock in rats by inhibiting HMGB1. Inflammation 31: 227-234, 2008.

45. Abraham E, Laterre PF, Garbino J, Pingleton S, Butler T, Dugernier T, Margolis B, Kudsk K, Zimmerli W, Anderson P, et al: Lenercept (p55 tumor necrosis factor receptor fusion protein) in severe sepsis and early septic shock: a randomized, double-blind, placebo-controlled, multicenter phase III trial with 1,342 patients. Crit Care Med 29: 503-510, 2001

46. Fisher CJ Jr, Dhainaut JF, Opal SM, Pribble JP, Balk RA, Slotman GJ, Iberti TJ, Rackow EC, Shapiro MJ, Greenman RL, et al: Recombinant human interleukin 1 receptor antagonist in the treatment of patients with sepsis syndrome. Results from a randomized, double-blind, placebo-controlled trial. Phase III rhIL-1ra Sepsis Syndrome Study Group. JAMA 271: 1836-1843, 1994.

47. Cauwels A: Nitric oxide in shock. Kidney Int 72: 557-565, 2007.

48. Hagiwara S, Iwasaka H, Hidaka S, Hasegawa A and Noguchi $\mathrm{T}$ : Neutrophil elastase inhibitor (sivelestat) reduces the levels of inflammatory mediators by inhibiting NF-kB. Inflamm Res 58: 198-203, 2009.

49. Toda Y, Takahashi T, Maeshima K, Shimizu H, Inoue K, Morimatsu H, Omori E, Takeuchi M, Akagi R and Morita K: A neutrophil elastase inhibitor, sivelestat, ameliorates lung injury after hemorrhagic shock in rats. Int J Mol Med 19: 237-243, 2007. 
50. He Z, Zhu Y and Jiang H: Inhibiting toll-like receptor 4 signaling ameliorates pulmonary fibrosis during acute lung injury induced by lipopolysaccharide: an experimental study. Respir Res 10: $126,2009$.

51. Kim TH, Kim SJ and Lee SM: Stimulation of the $\alpha 7$ nicotinic acetylcholine receptor protects against sepsis by inhibiting toll-like receptor via phosphoinositide 3-kinase activation. J Infect Dis 209: 1668-1677, 2014

52. Li C, Hua F, Ha T, Singh K, Lu C, Kalbfleisch J, Breuel KF, Ford T, Kao RL, Gao M, et al: Activation of myocardial phosphoinositide-3-kinase $\mathrm{p} 110 \alpha$ ameliorates cardiac dysfunction and improves survival in polymicrobial sepsis. PLoS One 7: e44712, 2012.
53. Sadhu C, Dick K, Tino WT and Staunton DE: Selective role of PI3K delta in neutrophil inflammatory responses. Biochem Biophys Res Commun 308: 764-769, 2003.

54. Araki Y, Matsumiya M, Matsuura T, Kaibori M, Okumura T, Nishizawa $M$ and Kwon AH: Sivelestat suppresses iNOS gene expression in proinflammatory cytokine-stimulated hepatocytes. Dig Dis Sci 56: 1672-1681, 2011.

55. Wu F, Zhang W, Li L, Zheng F, Shao X, Zhou J and Li H: Inhibitory effects of honokiol on lipopolysaccharide-induced cellular responses and signaling events in human renal mesangial cells. Eur J Pharmacol 654: 117-121, 2011. 